\title{
Buried Structure in Block Copolymer Films Revealed by Soft X-Ray Reflectivity
}

Daniel F. Sunday ${ }^{1 *}$, Jacob L. Thelen ${ }^{1}$, Chun Zhou², Jiaxing Ren², Paul. F. Nealey², R. Joseph Kline ${ }^{1}$

1 Materials Science and Engineering Division, National Institute of Standards and Technology, 100 Bureau Drive, Gaithersburg, MD 20899

2 Institute for Molecular Engineering, University of Chicago, 5801 S Ellis Ave, Chicago, IL 60637

*Daniel.Sunday@nist.gov

Supporting Information 

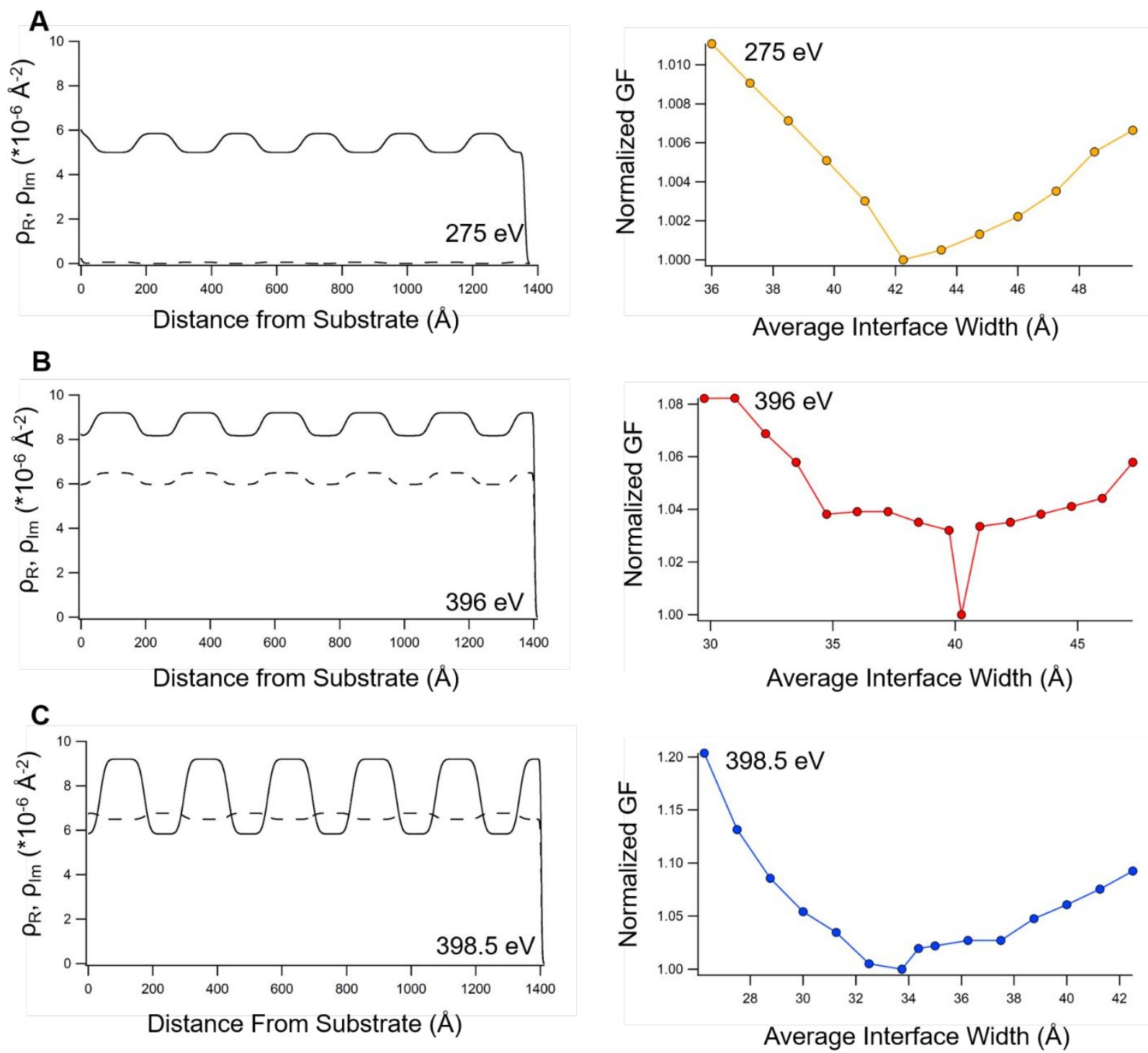

Figure S1: Left column shows scattering length density $(\rho)$ profiles for the $5.5 L_{0} \mathrm{PS}_{25}-b-\mathrm{P}_{2} \mathrm{VP}_{25}$ sample at $275 \mathrm{eV}, 396 \mathrm{eV}$ and $398.5 \mathrm{eV}$. Solid lines indicate the real component $\left(\rho_{\mathrm{R}}\right)$, dashed lines indicate the imaginary component $\left(\rho_{\mathrm{Im}}\right)$. Right column shows normalized goodness of fits (GF) as a function of the interface width around the best fit. Normalized GF is defined as the GF value at that interface width compared to the best overall GF.

Table S1: Chi-Squared values for best fits from the models where all interfaces were constrained to be identical and model with monotonic decay in the interface values.

\begin{tabular}{|c|c|c|}
\hline Energy (eV) & Identical Interface width & Decaying Interface Width \\
\hline 275 & 9.93 & 10.14 \\
\hline 396 & 6.56 & 6.73 \\
\hline 398 & 11.79 & 11.99 \\
\hline
\end{tabular}

Table S2: Interface widths as a function of $L_{\mathrm{o}}$ evaluated using the uniform width model 


\begin{tabular}{|c|c|c|}
\hline Sample & Sample Thickness & Interface width $(\AA)$ \\
\hline $\mathrm{PS}_{25}-b-\mathrm{P} 2 \mathrm{VP}_{25}$ & $0.5 L_{0}$ & $45 \pm 4.5$ \\
\hline $\mathrm{PS}_{25}-b-\mathrm{P} 2 \mathrm{VP}_{25}$ & $1.5 L_{0}$ & $48.3 \pm 1.25$ \\
\hline $\mathrm{PS}_{25}-b-\mathrm{P} 2 \mathrm{VP}_{25}$ & $2.5 L_{\circ}$ & $41.2 \pm 0.89$ \\
\hline $\mathrm{PS}_{25}-b-\mathrm{P} 2 \mathrm{VP}_{25}$ & $3.5 L_{0}$ & $37.2 \pm 0.87$ \\
\hline $\mathrm{PS}_{25}-b-\mathrm{P} 2 \mathrm{VP}_{25}$ & $4.5 L_{\circ}$ & $36.2 \pm 0.65$ \\
\hline $\mathrm{PS}_{102}-b-\mathrm{P} 2 \mathrm{VP}_{97}$ & $0.5 L_{\circ}$ & $44.5 \pm 5.7$ \\
\hline $\mathrm{PS}_{102}-b-\mathrm{P} 2 \mathrm{VP}_{97}$ & $1.5 L_{\circ}$ & $40.8 \pm 0.9$ \\
\hline $\mathrm{PS}_{102}-b-\mathrm{P} 2 \mathrm{VP}_{97}$ & $2.5 L_{\circ}$ & $36.05 \pm 0.6$ \\
\hline $\mathrm{PS}_{102}-b-\mathrm{P} 2 \mathrm{VP}_{97}$ & $3.5 L_{0}$ & $35.8 \pm 0.6$ \\
\hline
\end{tabular}

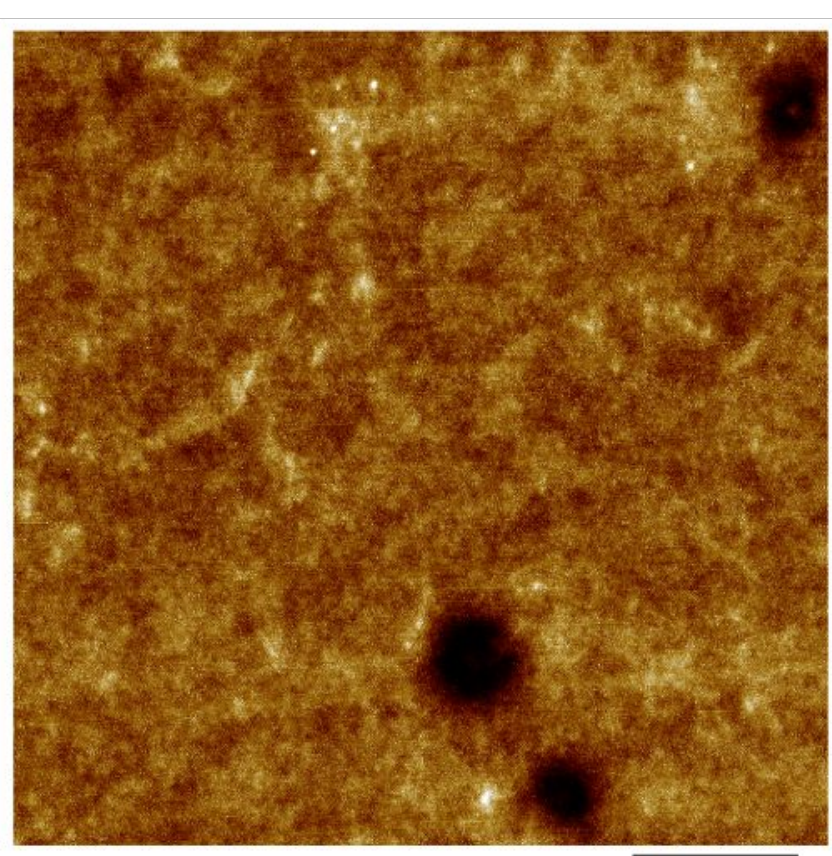

A

$2.6 \mathrm{~nm}$

$\overline{1.0 \mu \mathrm{m}}$

$-3.4 \mathrm{~nm}$

B

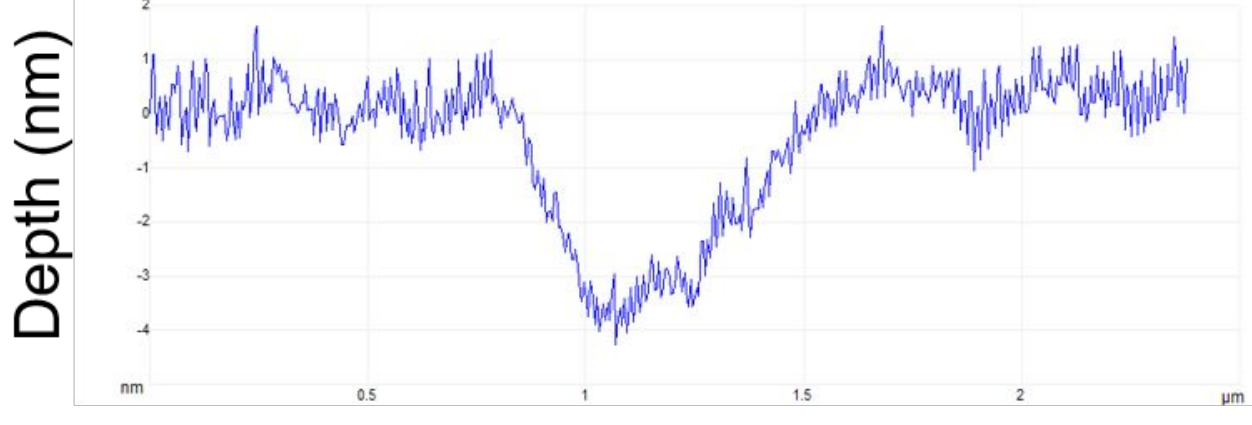

Width $(\mu \mathrm{m})$

Figure S2: AFM image of $\mathrm{PS}_{25}-b-\mathrm{P} 2 \mathrm{VP}_{25} 0.5 L_{0}$ film. 

A
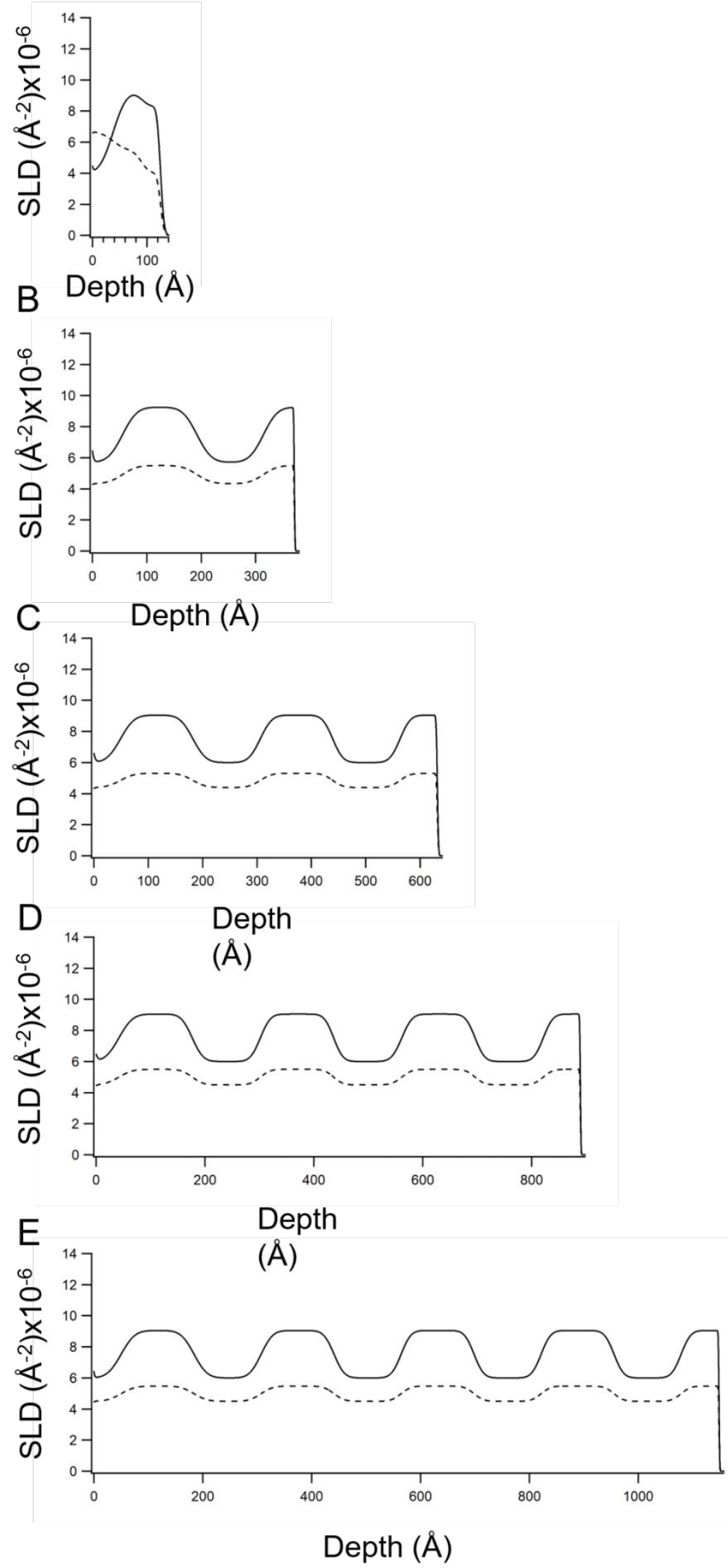
Figure S3: SLD profiles of $\mathrm{PS}_{25}-b-\mathrm{P} 2 \mathrm{VP}_{25}$ (reflectivity shown in Figure $3 A$ ) at $0.5 L_{\circ}(\mathrm{A}), 1.5 L_{\mathrm{o}}$ (B), $2.5 L_{\circ}(C), 3.5 L_{o}(D)$ and $4.5 L_{o}(E)$.
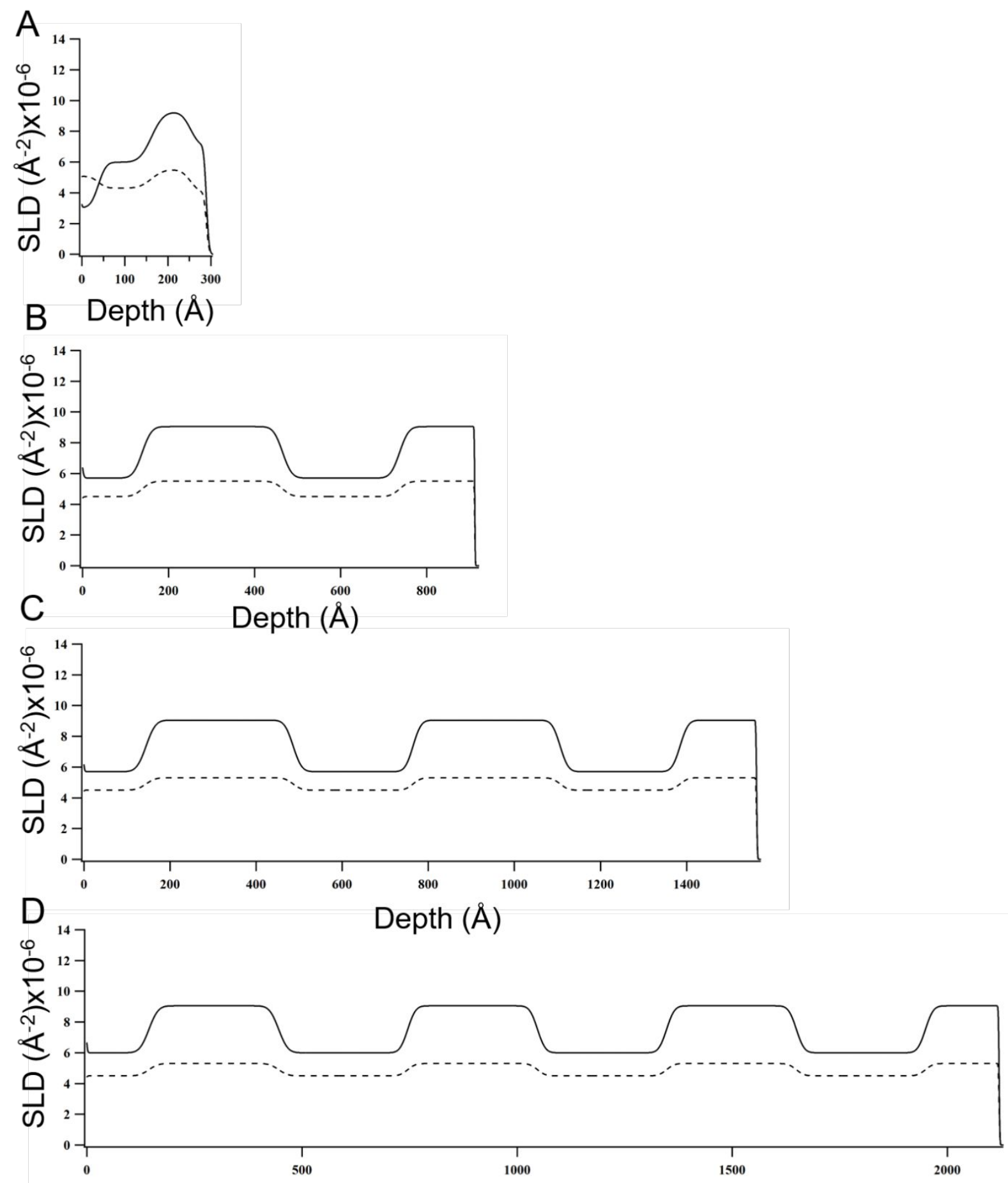

Depth $(\AA)$ 
Figure S4: SLD profiles of $\mathrm{PS}_{102}-b-\mathrm{P} 2 \mathrm{VP}_{97}$ (reflectivity shown in Figure $3 \mathrm{~B}$ ) at $0.5 L_{\circ}(\mathrm{A}), 1.5 L_{\circ}$ (B), $2.5 L_{\circ}(C), 3.5 L_{\circ}(D)$.

\section{Fitting $0.5 L_{0}$ PS-b-P2VP Samples}

Examining the fits to the $0.5 L_{o}$ PS-b-P2VP samples reveals additional information about the structure of the BCP near the substrate. Initially these samples were modeled with a two-layer fit where the optical constants of PS and P2VP were constrained to match the isotropic optical constants for those two materials at $398.5 \mathrm{eV}$. The result of this fit is shown in Figure S5, with the corresponding optical constant profiles shown in Figure $\mathrm{S} 6$ for both molecular masses. This model was unable to capture the minima of the lower $Q$ fringes. AFM images (Figure S2) reveal a small amount of surface topography in these films, and an added layer at the top surface with lower density (consistent with 3-4 $\mathrm{nm}$ deep holes) resulted in an improved fit to the system, but one that still did not fully capture the minima in the experimental data. In addition to being sensitive to composition, soft X-rays are sensitive to the orientation of the bonds at resonant energies. These measurements were conducted at $398.5 \mathrm{eV}$, where the $1 \mathrm{~s} \rightarrow \pi^{*}$ transition for the nitrogen atom in the pyridine ring occurs. The transition dipole for the pyridine ring is illustrated in the schematic in Figure $8 \mathrm{C}$, where a pyridine ring is oriented perpendicular to the substrate. Models were tested to explore the possibility that preferential molecular orientation of the pyridine ring results in changes in the optical constant profile from the isotropic model. As mentioned earlier, the magnitude of the shift in the optical constants is a function of the energy, functional group concentration and orientation. When the bond orientation is isotropic the impact of the bond orientation on the contrast can be neglected, but near interfaces the potential for preferential bond orientation increases. The sample was measured with an S polarized beam, so regardless of the incident angle the electric field vector will be parallel to the substrate. Preferential orientation of the aromatic ring perpendicular to the substrate will result in alignment of some fraction of the 
pyridine group with the electric field vector, resulting in a larger absorbance and greater shift of the optical constants relative to an isotropic sample. On the other hand, orientation of the pyridine ring parallel to the substrate will result in reduced absorbance. The optical constants were allowed to vary near the $\mathrm{SiO}_{2}$ substrate, and both samples showed an $\sim 4 \mathrm{~nm}$ thick layer where the real component decreases, and the imaginary component increases. This direction of the shift is consistent with the average orientation of the pyridine ring being perpendicular to the substrate. Without additional P polarized measurements the result cannot be confirmed, i.e. the change cannot be uniquely attributed to differences in molecular orientation. 
A
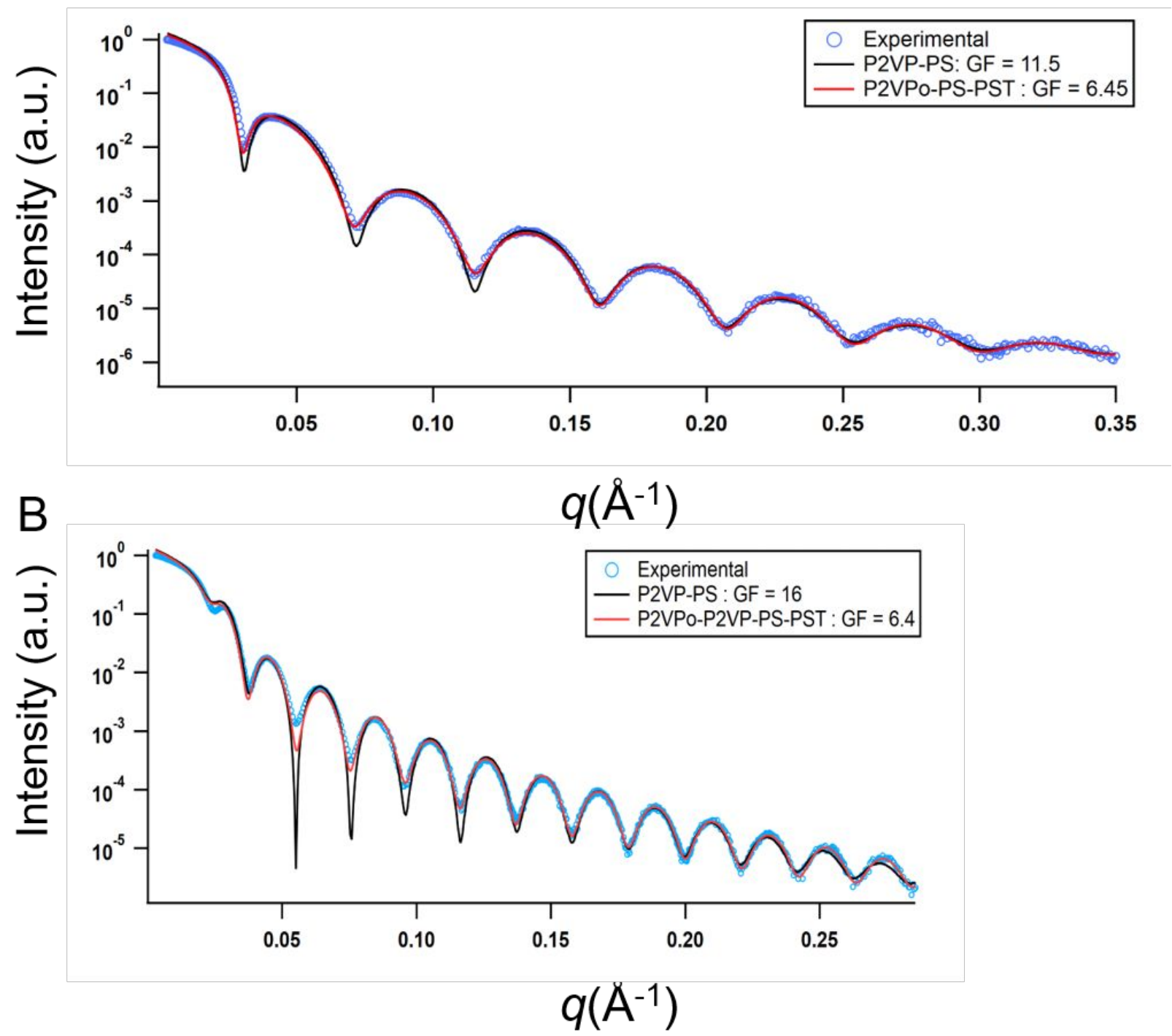

Figure S5: Experimental data and fits for the $0.5 L_{0}$ samples for $(A) P_{25}-b-P 2 V P_{25}$ and (B) $P S_{102^{-}}$ $b$-P2 $\mathrm{VP}_{97}$ measured at $398.5 \mathrm{eV}$. Fits are shown from simple two-layer models with optical constants for both layers equivalent to the bulk optical constants for PS and P2VP (-) and a model which captures the surface topography (layer labeled as PST) observed in AFM images (Figure S2) and preferential orientation of the pyridine ring (layer labeled P2VPo) near the SiOn substrate. For comparison of the changes in fit quality the goodness of fit $(G F)$ is also shown in the figure legend. The corresponding optical constant profiles are shown in Figure S6. Additional fits showing fits with just topography or orientation are shown in Figure S7. 

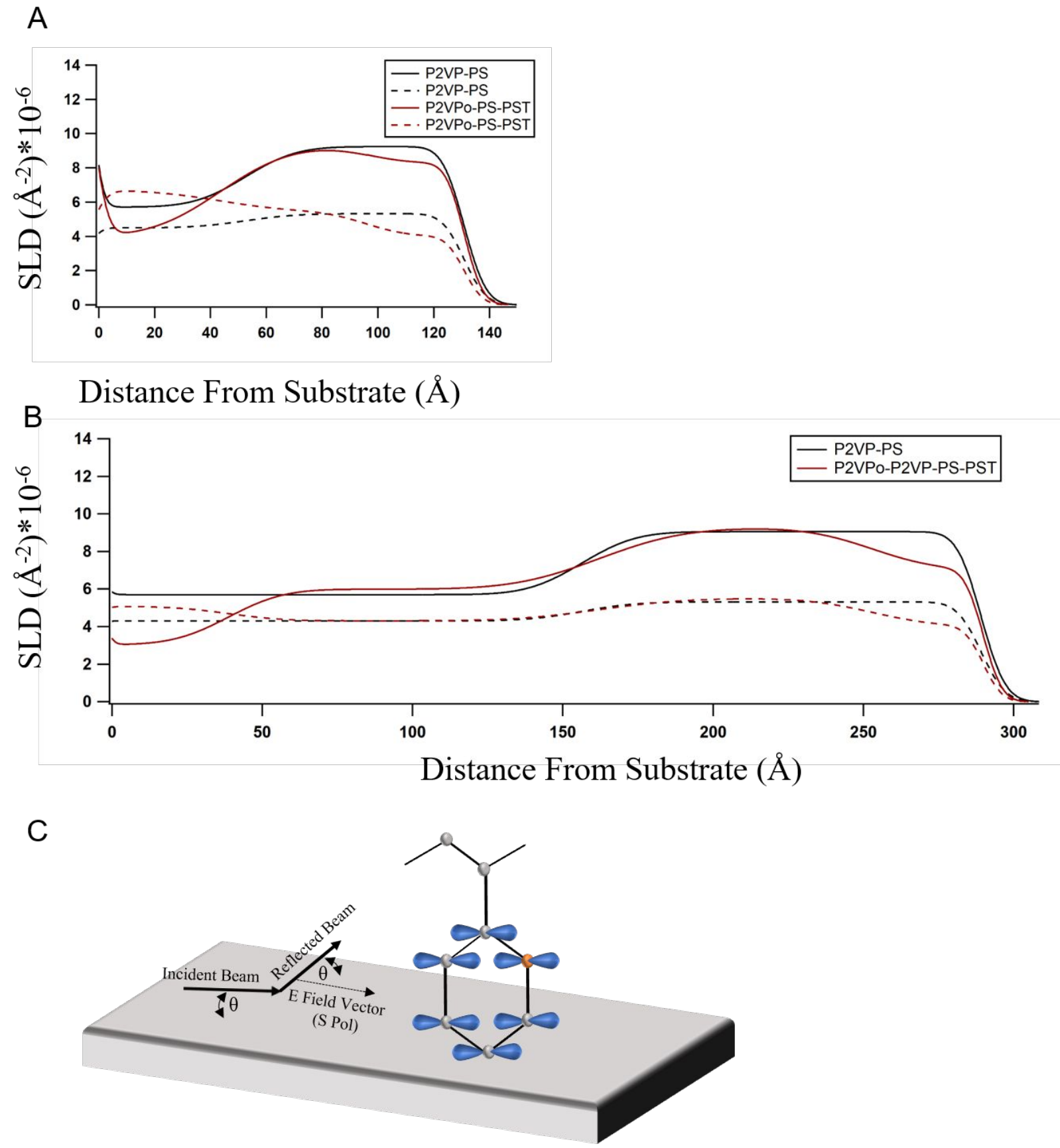

Figure S6: Optical constant profiles for $0.5 L_{\circ}$ samples of (A) $\mathrm{PS}_{25}-b-\mathrm{P}_{2} \mathrm{VP}_{25}$ and (B) $\mathrm{PS}_{102}-b-$ $\mathrm{P}_{2} \mathrm{VP}_{97}$ corresponding to the fits highlighted in Figure S5. Solid lines correspond to the real component, dashed lines to the imaginary component. The decrease in the both the real and imaginary components over the top $\approx 4 \mathrm{~nm}$ originates from surface topography, the change near the substrate is likely due to orientation of the pyridine ring. C) Schematic of the incident electric field vector for an s-polarized beam relative to a pyridine ring oriented perpendicular to the substrate. 

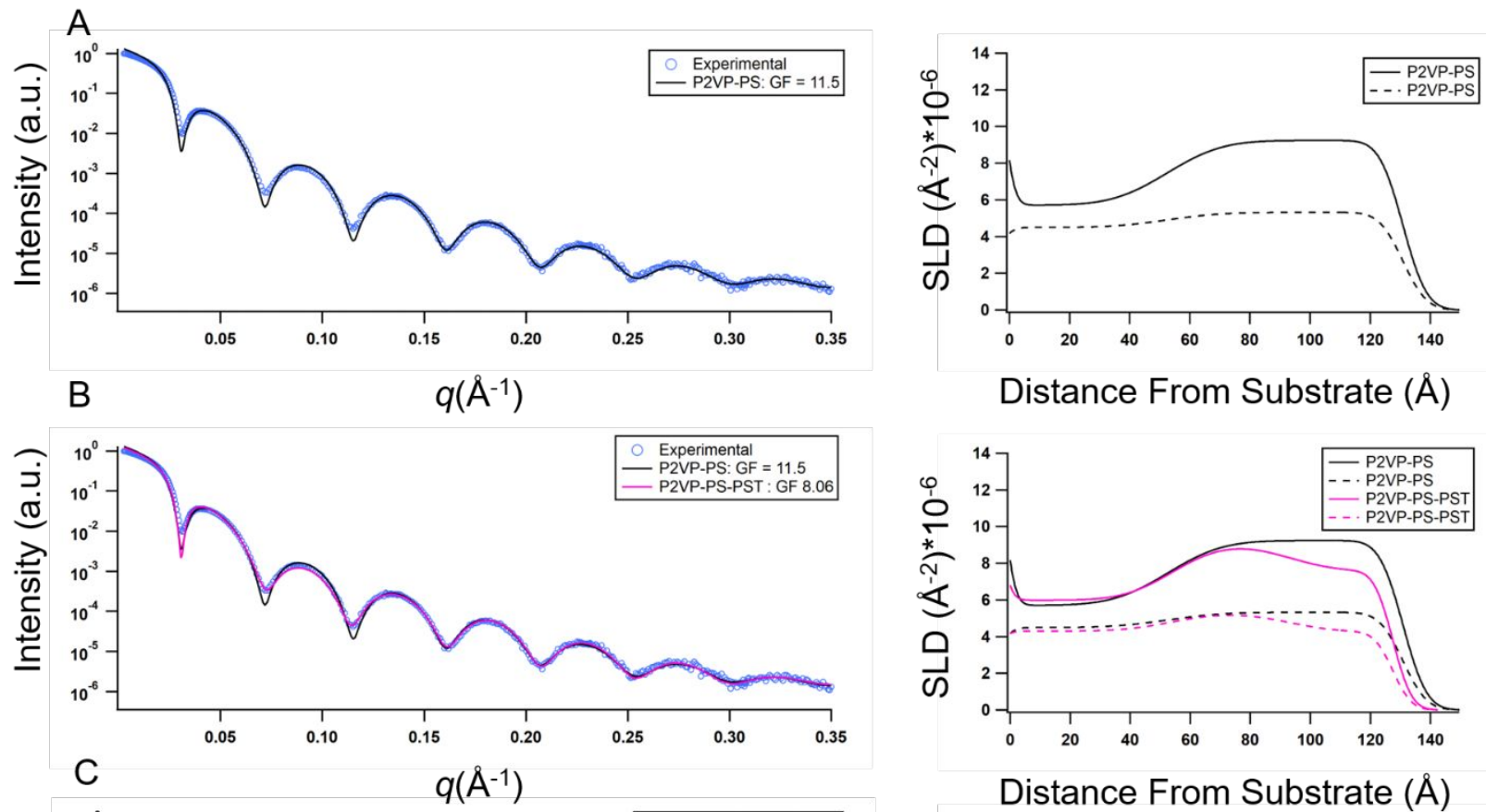

Distance From Substrate $(\AA)$
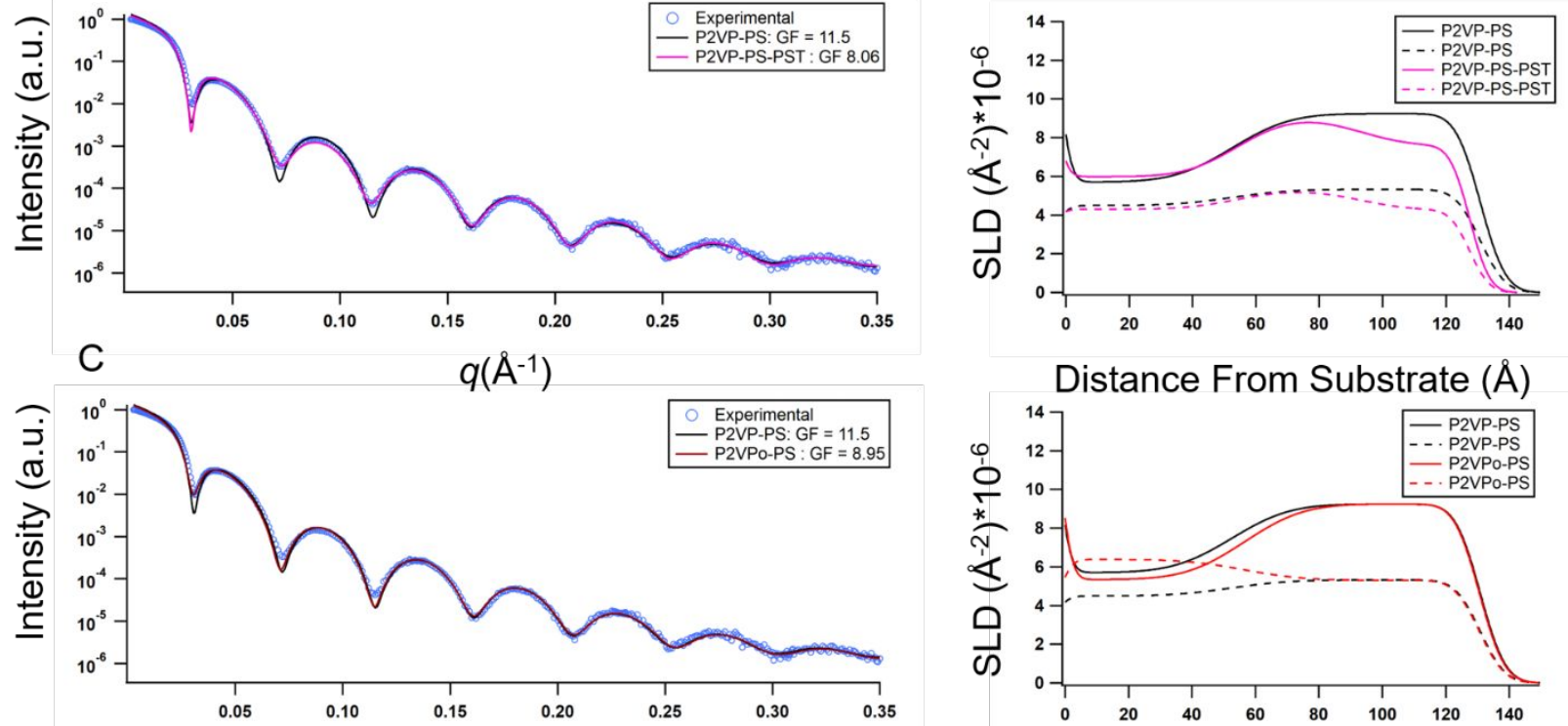

Distance From Substrate $(\AA)$
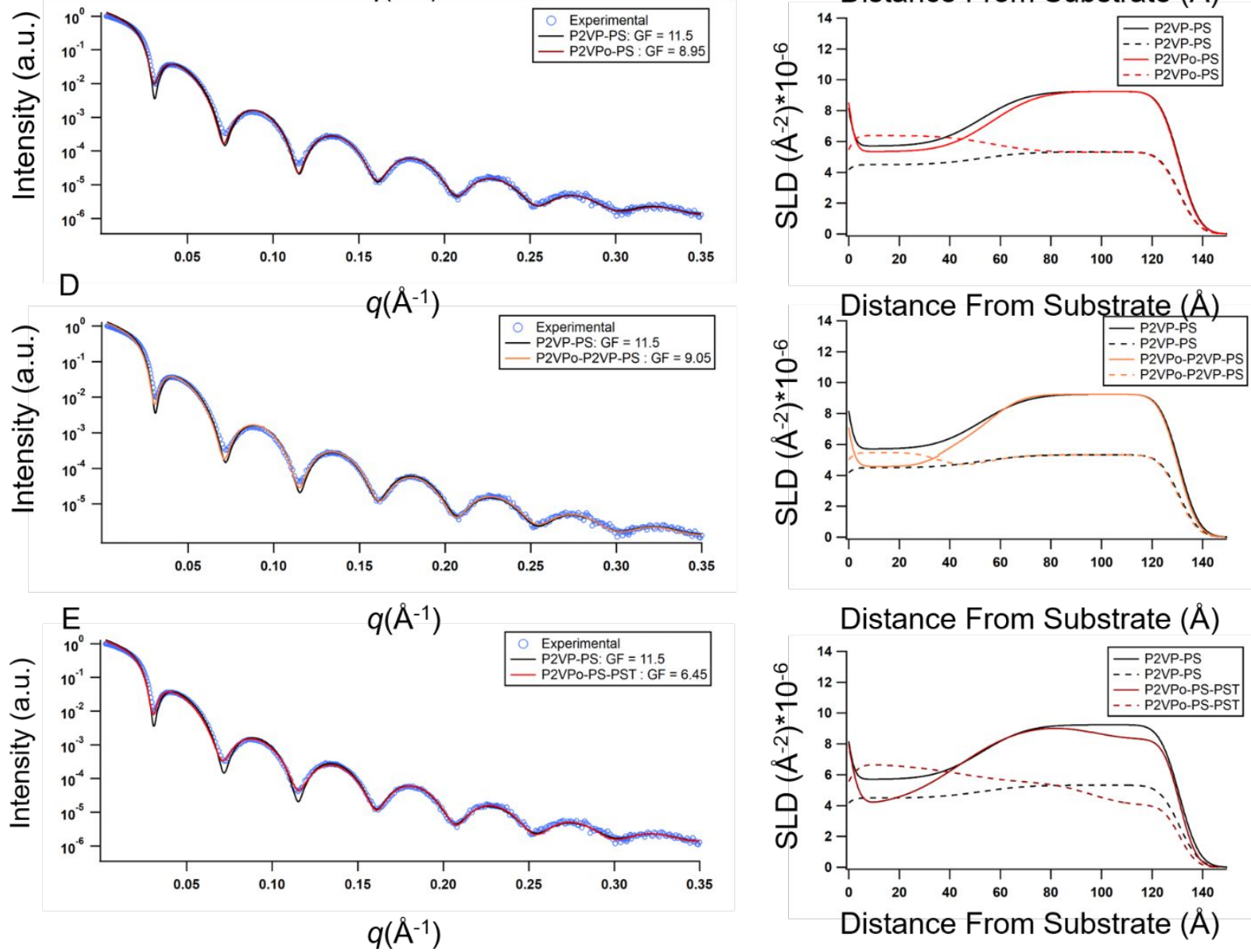

Distance From Substrate $(\AA)$

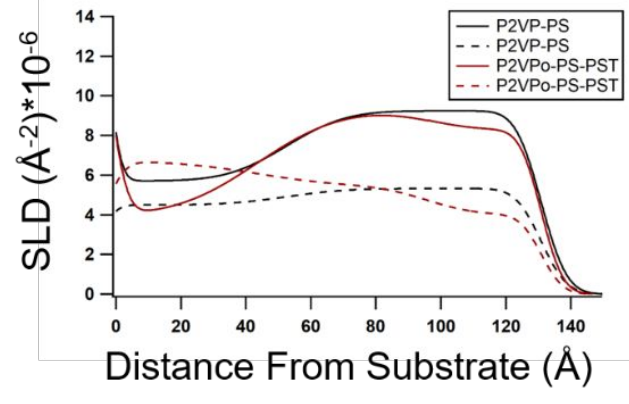

Figure S7: Fits to $0.5 L_{0}$ samples for $\mathrm{PS}_{25}-b-\mathrm{P}_{2} \mathrm{VP}_{25}$ as a function of model complexity. Comparisons with the reflectivity and SLD profiles of the 2 layer model are shown for parts B-E. 
The fits using the two-layer model are shown in (A). The fits with surface topography are shown in (B). Fits with an oriented layer of P2VP near the substrate are shown in (C). Fits using both an oriented layer of P2VP, a thin un-oriented layer of P2VP and a PS layer are shown in (D). Fits using an oriented layer of P2VP and topography in the PS layer are shown in (E).

\section{Measurements of a Triblock Polymer}

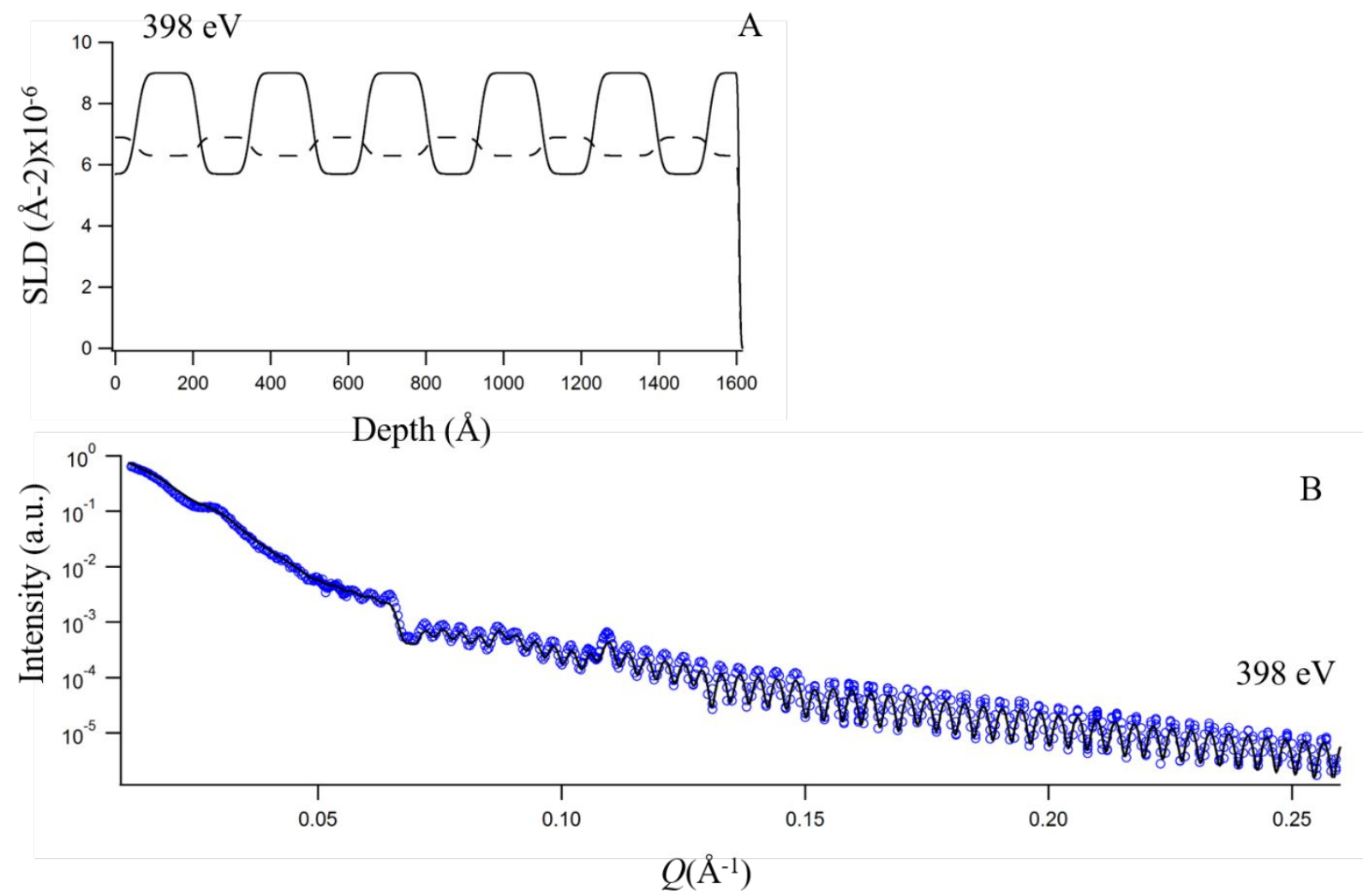

Figure S8: A) SLD profile for the best fit to the reflectivity curve measured at $398 \mathrm{eV}$ for the triblock. B) Measured reflectivity $(\circ)$ and best fit (solid line) to reflectivity data collected at $398 \mathrm{eV}$ on the triblock sample.

\section{Comparison of Variation in Lamellar Thickness vs Roughness}

Another structural variation that was considered in the model was the possibility that the lamellar thickness varied in a systematic way. In a reflectivity measurement thickness variations manifest in terms of changes in the location and magnitude of the fringes, while changes to the 
roughness result in variation in the fringe damping as a function of $q$. To demonstrate how those different signatures would appear in a reflectivity profile a set of simulations were conducted where the thickness was varied and compared to a simulation where the roughness was varied. The reflectivity from a baseline model where the thickness of the PS and P2VP layers were identical in each repeat unit was simulated and then two variations were considered (1) where the thickness of the bottom PS and P2VP repeat unit was $5 \%$ thicker than the rest of the lamellar and (2) where the bottom repeat unit was 5\% thicker and the top repeat unit was $5 \%$ thinner, so that the total thickness was identical to the baseline model. The results of these simulations are shown in Figure S9. To highlight the difference between changes in lamellar thickness compared to changes in interfacial roughness another simulation was conducted where the baseline model was compared to a model with identical thickness, but different interface widths, these results are shown in Figure S10.

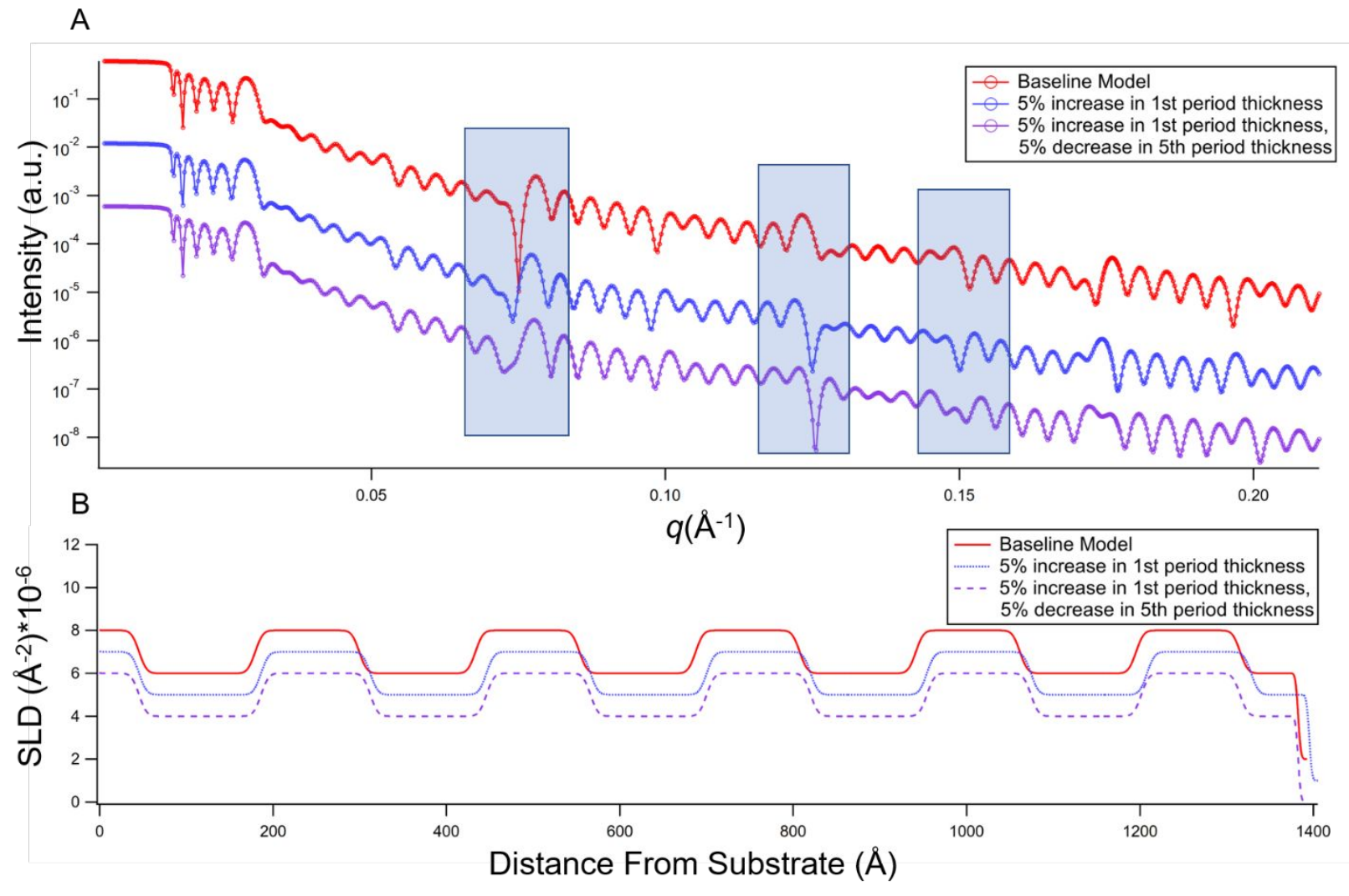


Figure S9: A) Reflectivity from 3 model systems, comparing the impact of changes in the lamellar thickness. The boxes highlight positions where there are clear differences in the reflectivity profiles, although these are not the only regions where there are changes B) Corresponding model systems for the SLD profiles. The red and blue profiles are shifted vertically for clarity by 2 ${ }^{*} 10^{-6} \AA^{-2}$ and $1^{*} 10^{-6} \AA^{-2}$, respectively. In this case only the real component is shown.

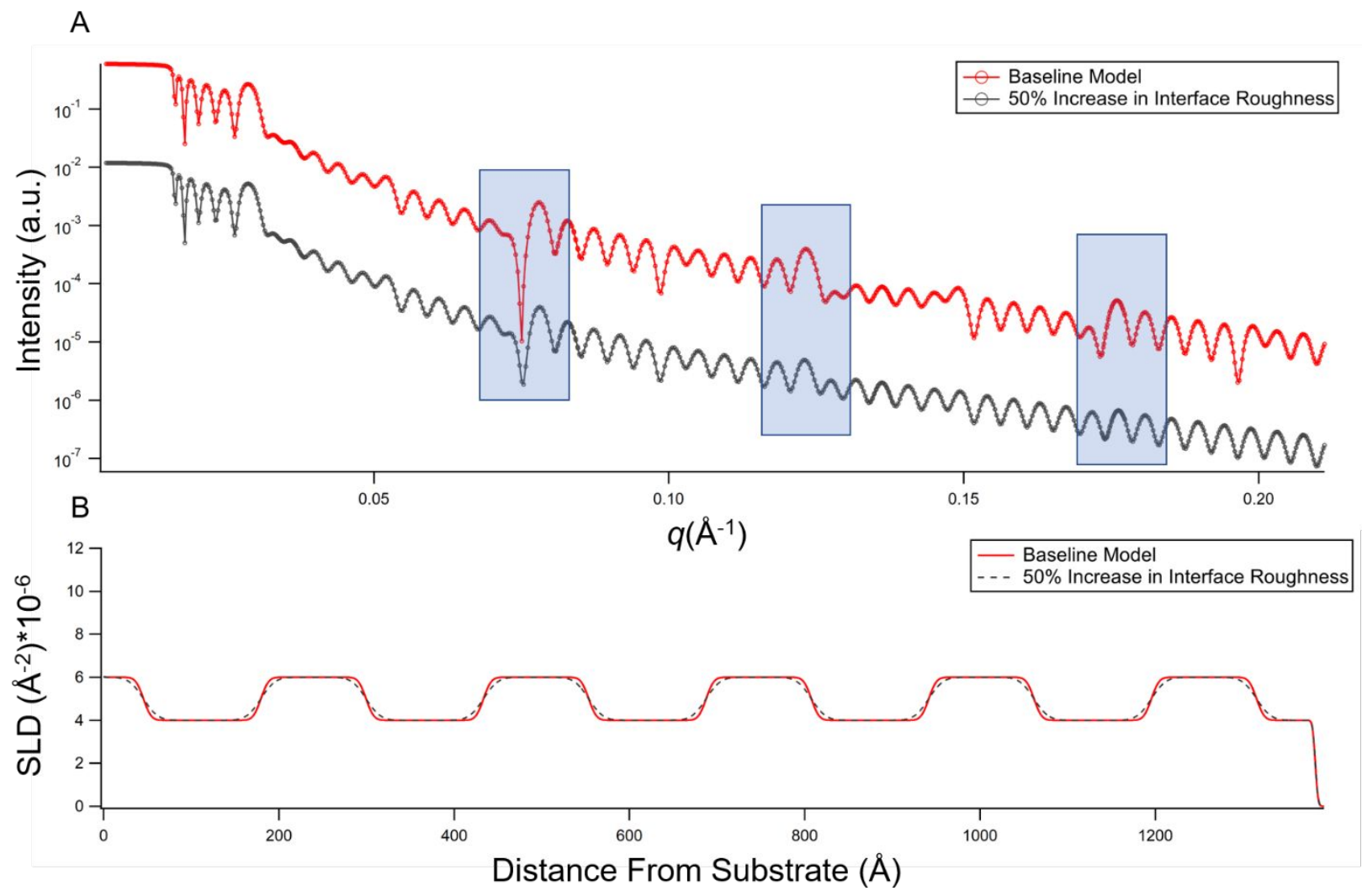

Figure S10: A) Reflectivity between two model systems with different interface widths, but identical layer thickness. B) Corresponding SLD profiles, showing on the real component for visual clarity. 